Voix et Images

voixetimages

\title{
Quatre enfants et un fantôme
}

\section{Michel Biron}

Volume 25, numéro 1 (73), automne 1999

Rêver l'enfance : Littérature et psychanalyse

URI : https://id.erudit.org/iderudit/201469ar

DOI : https://doi.org/10.7202/201469ar

Aller au sommaire du numéro

\section{Éditeur(s)}

Université du Québec à Montréal

\section{ISSN}

0318-9201 (imprimé)

1705-933X (numérique)

Découvrir la revue

\section{Citer cet article}

Biron, M. (1999). Quatre enfants et un fantôme. Voix et Images, 25(1), 193-199.

https://doi.org/10.7202/201469ar d'utilisation que vous pouvez consulter en ligne.

https://apropos.erudit.org/fr/usagers/politique-dutilisation/ 


\section{Roman}

\section{Quatre enfants et un fantôme}

\section{Michel Biron, Université du Québec à Montréal}

Voici cinq livres fort différents qui ont au moins deux choses en commun: tous sont écrits de façon extrêmement soignée, dans une langue non seulement maîtrisée, mais juste, personnelle, une langue d'écrivain. S'agit-il pour autant d'une langue de romancier? Trois de ces textes portent le sous-titre "récit" ou "récits", comme s'ils satisfaisaient ainsi à des exigences moins lourdes, moins encombrantes que celles du roman (ou de la nouvelle). Les deux autres se présentent comme des romans, mais on verra qu'ils résistent, eux aussi, au romanesque. Les personnages que ces récits ou ces romans, peu importe comment ils se désignent eux-mêmes, mettent en scène ne sont jamais des personnages d'emblée romanesques: c'est même leur pauvreté de personnage, leur indifférence, voire leur hostilité envers l'univers grandiose du roman qui les définit au bout du compte. Avec un succès inégal, les cinq auteurs trébuchent ainsi sur le même obstacle - de façon avouée dans certains cas, en faisant mine de rien dans les autres. Cette difficulté pourrait s'énoncer comme ceci : comment inventer un personnage proprement romanesque à partir de figures familières, prises dans le smog de souvenirs à la fois intimes et incertains, des figures reconnaissables, certes, mais comme oubliées déjà malgré le caractère autobiographique de plusieurs de ces récits, des figures presque irréelles tant elles se dérobent à toute forme de symbolisation?

Publié dans la collection "Haute enfance" de Gallimard, Mille eaux ${ }^{1}$ d'Émile Ollivier raconte l'enfance haïtienne de l'écrivain-sociologue, une enfance placée sous le signe d'une injonction dont on ne se libère pas aisément: le père voulait absolument qu'Émile devienne écrivain. Il lui impose d'ailleurs le nom d'un écrivain français du $\mathrm{XIX}^{\mathrm{e}}$ siècle, qui fut député et ministre de la Guerre en 1870. L'héritage est lourd, mais le père quitte bientôt la maison familiale pour mourir peu de temps après. Élevé par sa mère loin du monde paternel (Émile n'apprendra qu'au moment des funérailles que son père a eu neuf filles de neuf mères différentes), le jeune Émile retrouvera ce dernier à travers l'écriture.

La belle image qui donne son titre à ce récit de souvenirs, Mille eaux, diminutif d'Émile Ollivier, renvoie à un passé multicolore et discontinu. Les souvenirs d'enfance s'ordonnent moins autour d'une séquence d'événements chronologiques qu'au hasard de l'anamnèse, comme si les faits et les personnages convoqués par la mémoire de l'écrivain formaient une sorte d'archipel, 
vaste univers où l'effet d'ensemble ne se perçoit que de loin. L'autobiographie tient presque toujours de la sociographie, l'anecdote la plus simple révélant toute une façon d'être en société. Le conflit social ne se situe pas ici à l'échelle des groupes, mais dans l'intimité domestique, comme dans ce passage où le narrateur court annoncer la mort de Madame Choisil à sa mère et lui lance, tout essoufflé : "Maman! Maman! Madame Choisil est mort!" Sa mère le regarde, découragée, et lui répond durement, sans même penser à son amie qui vient de mourir: "Madame Choisil est mor-te, imbécile!" (p. 59)

"J'ai toutes les peines du monde à ordonner mes souvenirs d'enfance " (p. 48), écrit le narrateur. Ce sont des images, des sensations, quelques figures originales et saisissantes, comme cette Madame Gano, métisse dans la quarantaine qui se promène nue devant le petit garçon de 10 ans, ou encore cet adolescent tué par son ami d'une balle dans la poitrine malgré le talisman qui, croyait-il, devait le protéger. Le fil chronologique n'est pas invisible, mais il s'entortille sur lui-même, laissant apparaître d'autres fils que le lecteur est invité à suivre. Tout le récit se déroule finalement hors champ; loin de la scène sociohistorique où ont lieu des drames collectifs, non pas parce que le social passe au second plan, mais parce qu'il ne se révèle jamais mieux que dans ces images secrètes, liées au destin de l'individu.

Si les réminiscences commencent par le père, à la fin il n'y en a plus que pour la mère, cette mère "aux pieds poudrés." qui, folle à lier selon le voisinage, communiquera à son fils un certain bonheur de la migra- tion. Cette tentation de la route. n'a pas commencé le jour où il a quitté Haïti, mais. bien avant, dès son enfance en fait: "Je suis donc fils de migrants et, en ce sens, ma migration ne date point d'hier. Mes veines sont irriguées des globules rouges de l'errance. Et j'ajoute la plupart du temps: par-dessus le marché, ma mère avait les pieds poudrés de la poussière des chemins." (p. 142-143) Faut-il venir de Port-au-Prince pour éprouver ainsi une telle légèreté du départ, une telle absence de tragique, en dépit des horreurs dont le sujet garde la mémoire? Car l'écrivain de soixante ans n'oublie rien de cette ville, confondue à jamais avec son enfance et avec les mille eaux sur lesquelles s'ouvre celle-ci. Ses souvenirs appartiennent à ce lieu, mais ne se fixent jamais sur lui : ils sont écrits dans le bonheur désordonné d'un récit fait de mille digressions.

$$
* *
$$

Le second roman de Jacques Marchand, Les vents dominants ${ }^{2}$, aurait pu appartenir à la même collection que celui. d'Ollivier, puisque le narrateur, Guillaume, revient sur les lieux de son enfance, à Montréal. Mais la "haute enfance" n'affleure chez lui qu'à travers le présent, prófondément dissimulée dans un roman familial assez compliqué. Guillaume étudie dans des laboratoires universitaires de Seattle et habite chez Scott, son père (celuici exige que son fils l'appelle par son prénom). Scott vit avec Brenda, une ancienne danseuse peu sympathique et extrêmement possessive. Entre autres activités, il réalise des films pour la télévision. Tous trois se 
rendent à Montréal; où Scott avait déjà vécu afin d'éviter le service militaire. Ils logent chez Paule, une actrice qui fut la maîtresse de Scott quinze ans plus tôt et qui devient, à cette occasion, celle du fils. Quant à la mère de Guillaume, elle est partie depuis longtemps, à cause de Paule précisément, pratiquer la médecine quelque part en Afrique.

Le roman précédent de Marchand, Le premier mouvement ${ }^{3}$, se déroulait en Floride, autour de deux frères et avait une densité d'écriture remarquable, dénuée de prétention. Dans Les vents dominants, l'écriture n'a rien perdu de son tranchant, mais le roman n'a plus aucun mystère. L'insupportable ménage à quatre a quelque chose de dérisoire, tant les personnages sont pétris de narcissisme, à commencer par le narrateur luimême, qui ne cesse de se regarder écrire et de noter ses observations soi-disant personnelles dans une sorte de carnet intime qu'il laisse traîner un peu partout. Un jour, Guillaume s'aperçoit que Paule a fouillé dans ses écrits. Dès lors, la marmite explose: Brenda, qui éprouve depuis longtemps une passion à moitié incestueuse pour Guillaume, surprend ses ébats avec Paule. Le narrateur s'enfuit aussitôt dans la voiture de celle-ci et finit par abandonner le véhicule au bout d'un chemin forestier avant de s'enfoncer profondément dans la nature. Il n'en sort que quelques jours plus tard, en suivant la direction des vents dominants, vers l'est, et en retournant surtout vers la maison de Paule, où le ramènent d'autres vents plus dominants encore, ceux du désir. Scott et Brenda sont déjà repartis dans l'ouest, malgré leur légitime inquiétude, ce qui donne à Guillaume le champ libre pour l'acte final: il égorge Paule en pleine rue, en plein jour. La scène paraît extrêmement violente, mais le lecteur ne ressent rien de plus qu'une sorte de dégoût furtif, à l'instar des passants qui tournent la tête et continuent leur chemin. On est à peine ému pour la pauvre actrice, tant le passage à l'acte est raconté en catastrophe. Guillaume n'a pas tué sa maîtresse: il l'a tout bonnement zappée.

\section{$*^{* *}$}

L'enfance chez Diane-Monique Daviau a une tout autre couleur, un autre poids que l'enfance haïtienne d'Émile Ollivier. Il faut dire que la mère, dans Ma mère et Gainsbourg ${ }^{4}$, n'a rien d'attachant et ne laisse à sa fille aucun héritage, aucune image forte comme celle des pieds poudrés: elle ne connaît ni les joies ni la fatigue de l'errance. Son corps massif, écrasant, est rivé au sol, à jamais. Même morte, elle ne se décide pas à partir, à laisser sa fille tranquille. Qui fut-elle? On ne le sait pas trop. Elle aimait parler en société, mais c'était une personne fermée, incapable de tendresse ou de générosité, en particulier envers sa fille, qui le lui rendait bien. Effacée malgré son obésité, cette mère n'a jamais eu d'intériorité : elle est vide, simple papier carbone destiné à la reproduction et ne comprenant pas que sa fille ne se reproduise pas à son tour. "C'est bien simple, constate sa fille avec désespoir, c'est comme si elle n'avait jamais existé." (p. 26)

C'est donc la fille qui engendre la mère, pourrait-on dire en 
paraphrasant Ferron ${ }^{5}$. Son récit commence par la mort de sa mère et par l'impossibilité même de raconter une vie qui semble n'avoir pas eu lieu. Comment faire le deuil d'une absence, se demande-t-elle? Comment inventer sa propre mère, lui donner une identité, une présence, comment faire de cette mère fuyante un véritable personnage, une figure individuelle qui ne soit pas seulement un corps informe, privé de regard, totalement fermé aux autres et incapable de rire? La mère n'est même pas franchement détestable, ce qui, après tout, en aurait fait un personnage plus intéressant: elle n'est détestable que parce qu'elle est incapable d'aimer sa fille, elle n'est donc détestable que pour sa fille. Pour le reste du monde, elle n'est rien du tout. C'est pourquoi la narratrice lui adjoint Gainsbourg, qui est, lui, un personnage tout fabriqué, romanesque en diable, la figure antagoniste par excellence. Gainsbourg n'a d'autre fonction ici que de créer le plus violent des contrastes: à lui seul, il incarne toute une société - et c'est précisément cela qui fait défaut à la mère, toute repliée sur sa propre personne, privée de la référence à un monde extérieur qui ne serait pas le monde immédiat et, partant, étrangère à l'univers de l'écriture. D'une certaine manièré, elle rappelle toutes les mères de la littérature québécoise, mais en pire, une mère pitoyable et impardonnable.

Troué comme la robe de sa mère que la narratrice tient à conserver, le récit ne cesse de frôler le même centre absent, tournant autour du vide maternel avec un désespoir qui paraît souvent sans limites. Au-délà du manque, au-delà même de cette incapacité à ressentir la perte de sa mère, la narratrice découvre que sa tristesse, loin de s'apaiser, n'en devient que plus définitive: "Arrive un âge où la vie, c'est mourir. Toute la vie, c'est mourir. Mourir jour et nuit, mourir toutes les saisons, mourir pour rien, mourir longtemps, parents mourir, oncles, tantes mourir, collègues mourir, amis mourir. Sœur, frère mourir." (p. 74) Pas question, dans ce contexte, de laisser sa mère se déployer comme personnage, d'en faire un individu complexe, singulier. En dépit des quelques petites choses aimables qu'elle trouve à dire à son sujet, elle intervient pour l'empêcher d'exister autrement qu'à titre de mauvaise mère. Ce faisant, elle commet un péché romanesque, car elle tue le personnage dans l'œuf:

Si ce personnage se trouvait dans une de mes nouvelles, dans un roman, je ne sais si j'arriverais, finalement, à le laisser tel quel, intouchable, imprenable. Peutêtre que oui. Peut-être bien. Je pense à ce personnage de mère dans "Le cherche-étoiles": elle reste inaccessible jusqu'à la fin. La différence, c'est que le fils, lui, lâche prise, finit par renoncer à l'étreinte espérée. 'Moi, j'ai encore mal quand je pense au fait que ma mère ne m'a jamais. dit, ou clairement démontré, qu'elle m'aimait. (p. 120-121)

D'où le malaise du lecteur qui se voit contraint de prendre le parti de la fille contre le personnage même qu'elle souhaite inventer.

\section{$* *$}

Comment naît le malheur? On pourra s'en faire une idée en lișant le dernier roman d'Anne Hébert, Un 
babit de lumière ${ }^{6}$, qui raconte le drame d'une famille espagnole vivant à Paris. La mère, Rose-Alba Almevida, est concierge; le père, Pedro, ouvrier en bâtiment; le fils, Miguel, joue avec une poupée et aime les robes de sa mère, malgré les avertissements péremptoires du père. Après une scène violente durant laquelle son père découvre sa poupée et la réduit furieusement en mille morceaux, Miguel décide de s'enfuir, de quitter ce monde trop viril, de fuir un père qui n'est pour lui qu'un étranger. Alors que la mère et le fils se composent un univers de secrets, le père se présente comme l'expression parfaite, presque caricaturale, du machisme le plus rude: "Je suis le Père, le Mari, le Pater Familias" "(p. 36), se dit-il plus d'une fois. Maitre de la maison, maître du corps de sa femme, il devient fou de rage lorsqu'il l'aperçoit transformée en blonde. Pourtant, elle croyait lui faire plaisir et elle avait même tout donné ce qu'elle possédait - jusqu'à son alliance - pour cette simple coquetterie. Son mari la soupçonne alors du pire et, plus dégoûté encore de sa nouvelle allure que du mensonge qu'elle avait inventé pour justifier la perte de l'alliance, il rêve de la tuer.

De retour à la maison, Miguel voit alors son père frapper sa mère et repart aussitôt dans les rues de Paris, où il rencontre un danseur, JeanEphrem de la Tour. Sa mère le surprend au spectacle de ce dernier, dans un endroit appelé le "Paradis perdu ". Comme son fils, elle assiste au spectacle et, peu à peu, elle prend goût à cet univers neuf, celui de la danse et du travestissement. Pendant ce temps, le père fait des haltères, comme pour préparer la vengeance promise. Au moment d'étrangler sa femme, il vacille toutefois, de peur que les voisins n'accourent, puis il disparaît à jamais de la maison, loin de Rose-Alba qui s'accroche ensuite au bras d'un riche client du "Paradis perdu ". Tous finissent par abandonner Miguel: son père étranger, sa mère transformée en furie et même Jean-Ephrem, chassé du "Paradis perdu". Comme dans les romans, il ne reste plus que la Seine, où Miguel se jette à la fin, en imaginant sa délivrance, une mort plus douce que l'enfance, une lumière enfin: "Quelqu'un de sacré, que je ne connais pas encore, me prépare en secret, au milieu des vagues et des frissons gris, un habit de lumière pour quand je serai arrivé parmi les morts." (p. 137)

Pour exposer le malheur de l'enfant, Anne Hébert a choisi un procédé déjà exploité dans Les fous de Bassan $^{8}$, celui de l'alternance des narrateurs. S'il n'est pas nouveau le roman épistolaire, au xviII ${ }^{\mathrm{e}}$ siècle, fonctionnait déjà suivant le même principe - ce procédé a l'avantage de créer une tension entre les différents points de vue, tous liés aux mêmes événements. Il exige toutefois que chaque personnage ait sa vision de l'histoire, son propre point de vue, ce qui n'est pas vraiment le cas du père, ni même du fils, car ce dernier est à la position de la victime ce que son père est à celle du bourreau. Le personnage qui domine tous les autres, en réalité, c'est celui de la mère, cette femme qu'on aperçoit, dès le seuil du roman, accoudée à sa fenêtre, fière de son corps, l'offrant au regard des passants ou des voisins, fière aussi de son nom qu'elle décline comme une incantation, 
“Rosa, Rosie, Rosita, rose d'Espagne brûlante et musquée, Rose-Albạ Almevida." (p. 12). Tous les autres personnages, le fils comme le père et même l'Ange des Ténèbres, JeanEphrem, ont le regard fixé sur cette furie. La fascination qu'elle suscite s'étend jusqu'à la voisine; Mme Guillou, double de l'auteure, qui observe de l'extérieur celle qui est à la fois la mère et la putain, celle par qui le déshonneur s'est installé dans la maison et qui constitue, à n'en pas douter, la véritable, la seule héroïne de.ce roman.

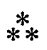

Certains morts font date: leur disparition est célébrée dans l'histoire comme l'événement qui entraîne tous les autres et qui, par là, en constitue l'emblème, le symbole privilégié. La mort de Louis XVI ou, plus près de nous, en 1975, la mort de Franco possèdent incontestablement une telle force emblématique. Y a-t-il, au Québec, un seul mort qui ait laissé une telle marque dans l'imaginaire collectif? Les morts, nous n'aimons pas ça: c'est de mauvais augure pour un peuple qui se souvient surtout des sinistres prophéties qu'on lui a répétées. S'il est un mort qui devrait pourtant signifier quelque chose, c'est Maurice Duplessis, dont le décès subit, en 1959, possède toutes les caractéristiques d'un événement majeur: avant, c'était la Grande Noirceur, après, la Révolution tranquille. Or, en dépit de ces circonstances idéales, faites sur mesure pour donner un poids historique à sa sortie, le premier ministre du Québec incarne, si l'on peut dire, un mort de faible envergure. Certes, il meurt, comme l'écrit Ducharme au début du Nez qui voque, "célèbre et célibataire ${ }^{9}$, mais une fois mort, c'est comme si, lui aussi, comme la mère de Monique Daviau, n'avait pas vraiment existé Ducharme l'abandonne d'ailleurs sur le seuil de son roman. Au lieu de révéler la formidable emprise du vivant sur le monde qu'il gouvernait, le mort en efface le souvenir et s'efface lui-même, personnage résolument secondaire, devenu soudainement irreprésentable, inimaginable, livré à l'abstraction du mythe.

Peut-on vraiment raconter la mort de Maurice Duplessis? C'est là tout le propos de Gilles Marcotte, dans le récit final qui donne son titre au recueil, La mort de Maurice Duplessis ${ }^{10}$. À l'origine de ce récit se devine un étonnement, suivi d'un défi. Comment se fait-il, tout d'abord, que la littérature ne se soit pas emparé déjà de ce sujet en apparence si romanesque? Tous se souviennent de ce qu'ils faisaient le jour de l'assassinat de John F. Kennedy, mais qui, au Québec, se rappelle la mort de Duplessis? Le symbole est bien là, mais creux, vide de traces. Pas tout à fait vide, en réalité: pour Marcotte comme pour plusieurs personnes qui ont connu le règne de Duplessis, les traces sont, au contraire, multiples, innombrables, inoubliables. Mais ce sont des souvenirs personnels liés à une atmosphère générale davantage qu'à' la personne de Duplessis. La mort de Franco tire son sens de la haine que le personnage a suscitée de son vivant. Or, la Grande Noirceur, pour beaucoup de Québécois, $y$ compris les intellectuels, ne fut noire que rétrospectivement et personne ne songerait aujourd'hui à déboulonner la statue de Duplessis 
devant le Parlement de Québec. "Je ne l'ai pas vraiment haï, détesté " (p. 151), écrit Marcotte. Difficile, sans l'aiguillon de la haine, d'éprouver un réel soulagement, une véritable libération à la mort du Chef.

Raconter la mort de Duplessis, c'est raconter une mort dépourvue de grandeur symbolique en cherchant à l'élever, par une attention extrême aux détails, aux circonstances, aux anecdotes, à la hauteur de la littérature. Autant l'avouer d'emblée: un tel pari est absolument impossible à tenir et cette impossibilité-là contient peutêtre, à elle seule, tout le malheur de la littérature québécoise. Les pages manquent ici pour développer une telle idée, qui a l'air d'une boutade, mais le récit de Marcotte invite justement à interroger directement cette impossibilité, à construire du sens et du récit dans cette impossibilité même, en posant la question ainsi : pourquoi l'histoire du mort le plus célèbre du Québec moderne se refuse-t-elle à l'invention romanesque?

Il est curieusement plus simple d'écrire un récit sous la forme d'un "Commentaire de l'Épître aux Romains "que de raconter la mort de Duplessis. Ou de croquer quelques visages d'inconnus, des touristes, un médiocre joueur de hockey, etc. Ou encore de raconter son "Autobiographie ". Dans tous ces cas, les personnages se donnent au récit sans résistance, dans la liberté de l'invention. Mais le narrateur est attiré précisément par ce qui lui résiste et il choisit, par le titre même du recueil qui donne au dernier récit une portée plus générale, de faire graviter tous ces personnages autour d'un centre creux, au plus près de ce fantôme éminent appelé Maurice Duplessis.

1. Émile Ollivier, Mille eaux, Paris, Gallimard, coll. "Haute enfance", 1999, 172 p.

2. Jacques Marchand, Les vents dominants, Montréal, l'Hexagone, coll. "Fictions ", $\mathrm{n}^{\circ} 108,1999,140 \mathrm{p}$.

3. Jacques Marchand, Le premier mouvement, Montréal, l'Hexagone, 1987, $90 \mathrm{p}$.

4. Diane-Monique Daviau, Ma mère et Gainsbourg, Québec, L'instant même, 1999, $183 \mathrm{p}$.

5. "C'est le Fils qui a engendré le Père", (Jacques Ferron, Le Saint-Élias, Montréal, Typo, 1993, p. 99).

6. Anne Hébert, Un babit de lumière, Paris, Seuil, 1999, $137 \mathrm{p}$.

7. Voir aussi plus loin: "C'est moi le mari, le père, le maître de maison. Je fais bouillir la marmite chez moi. Paye en poche, je rentre." (p. 46).

8. Anne Hébert, Les fous de Bassan, Paris, Seuil, 1982, 253 p.

9. Réjean Ducharme, Le nez qui voque, Paris, Gallimard, 1967, p. 10.

10. Gilles Marcotte, La mort de Maurice Duplessis et autres récits, Montréal, Boréal, 1999, 197 p. 